\title{
Salario y calidad de vida de los trabajadores de la comuna La Candelaria Medellín*
}

\section{Salary and quality of life of the workers of the commune La Candelaria Medellin}

\author{
DOI: https://doi.org/10.17981/econcuc.40.2.2019.06
}

Artículo de investigación. Fecha de recepción: 24/05/2019 Fecha de aceptación: 09/08/2019

John Freddy Castro Álvarez

Corporación Universitaria U de Colombia. (Medellín, Colombia)

investigaciones2@udecolombia.edu.co

\section{Claudia Marcela Londoño Atehortúa}

Arquidiócesis de Medellín. (Medellín, Colombia)

miangel112@hotmail.com

Para citar este artículo:

Castro, J. y Londoño, C. (2019). Salario y calidad de vida de los trabajadores de la comuna La Candelaria Medellín. Económicas CUC, 40(2), 87-100. DOI: http://dx.doi.org/10.17981/econcuc.40.2.2019.06

\section{Resumen}

Las variaciones del salario siempre han sido consideradas una de las mayores preocupaciones de los trabajadores, sin que estos se detengan a pensar en la capacidad de compra como garantía para una mejor calidad de vida, a pesar de que existen varios indicadores que se ocupan de este aspecto, como por ejemplo el índice de desarrollo humano, el cual tiene en cuenta la educación, la vivienda, la salud, etc. Esta indagación centro su análisis en el ingreso como elemento fundamental de esta condición para dar respuesta a la hipótesis planteada: "El 80\% de los trabajadores de la actividad de las confecciones de los estratos 2 y 3 de la comuna 10 de ciudad de Medellín (La Candelaria), trabajan en la informalidad laboral, con un salario bajo que no les permite tener una buena calidad de vida". El método que se utilizó para el desarrollo de esta investigación recoge aspectos metodológicos cualitativos y cuantitativos. En una primera fase se realiza un recorrido bibliográfico y documental, y en una segunda fase fue necesario tener en cuenta para la comprobación de la hipótesis planteada inicialmente, la recolección de datos estadísticos mediante una encuesta. Algunos de los resultados evidencian que para obtener un buen nivel de vida es necesario que otros miembros del hogar hagan su aporte. Se llegó a la conclusión de que el salario de los trabajadores es una variable clave que permite a los trabajadores de la actividad de las confecciones tener una buena calidad de vida.

Palabras clave: Salario real; calidad de vida; confecciones; comuna

Códigos JEL: A10, E12, B22

\begin{abstract}
Variations in wages have always been considered one of the biggest concerns of workers, without them stopping to think about purchasing power as a guarantee for a better quality of life, despite the fact that there are several indicators that address this aspect, such as the human development index, which takes into account education, housing, health, etc. This investigation focused its analysis on income as a fundamental element of this condition to answer the hypothesis raised: " $80 \%$ of the workers in the activity of clothing in strata 2 and 3 of commune 10 of the city of Medellín (La Candelaria), they work in informal employment, with a low salary that does not allow them to have a good quality of life." The method that was used for the development of this investigation includes qualitative and quantitative methodological aspects. In a first phase, a bibliographic and documentary tour is carried out, and in a second phase, it was necessary to take into account the statistical data collection through a survey to verify the hypothesis initially proposed. Some of the results show that to obtain a good standard of living it is necessary for other members of the household to make their contribution. It was concluded that the workers' salary is a key variable that allows workers in the clothing industry to have a good quality of life.
\end{abstract}

Keywords: Actual salary; quality of life; clothing; commune

* El proyecto fue financiado en su totalidad por la Corporación Universitaria U de Colombia. 


\section{INTRODUCCIÓN}

La sociedad desde sus orígenes se ha tratado de "hombres que se agrupan por exigencias de supervivencia frente a su medio ambiente, se unen para organizar y distribuir el trabajo o para establecer un mecanismo de control frente a los intereses antagónicos, y donde se sobrepone un grupo dominante" (Pérez, Hernández, Acosta y Chumaceiro, 2009, p. 274). Sin embargo, Villasmil y Crissien (2015) comentan que estas últimas décadas se "ha caracterizado por cambios vertiginosos desde perspectivas políticas, económicas, sociales y de mercado, que obligan emprender nuevas maneras de hacer las labores" (p. 246).

En tal sentido, la llegada de los años 90 trajo para la economía mundial grandes transformaciones, se pretendía con el cambio de modelo acabar con los desórdenes de carácter estructural que vivían la mayoría de las economías, especialmente las de américa latina. El neoliberalismo implementado, buscaba dar una mayor participación al mercado para disminuir el tamaño del estado, el laissez faire y laissez passer, fue el mecanismo inicial de ajuste para lograrlo, se pretendía acabar con todas esas barreras que impedían mejorar la dinámica en las negociaciones tanto internas como externas.

El nuevo escenario buscaba entre otros objetivos: aumentar el crecimiento económico, descargando esta responsabilidad en el fortalecimiento de las exportaciones y flexibilizando los precios a la baja, lo cual se lograría liberalizando las importaciones de bienes y servicios, con el fin de mejorar las condiciones de vida del consumidor final.

Se argumentaba a inicio de los años noventa, que aquellos países que se acostumbran a vivir con elevados niveles de inflación, reflejaban una industria poco competitiva, con alta capacidad ociosa instalada, sobre costos de producción que se trasmitirían vía precios al consumidor final; una consecuencia lógica de este proceso era el efectivo freno de las exportaciones afectando el crecimiento económico y la generación del empleo productivo; así mismo como la disminución en la capacidad de compra de los consumidores y como resultado final la disminución de la calidad de vida de los trabajadores.

Son precisamente estos cambios, como lo indican las autoras Hernández y Chumaceiro (2018), los detonantes de una crisis que busca satisfacer las necesidades y el aumento en calidad de vida de los trabajadores, es la búsqueda de esta calidad de vida la que genera la mayor lucha entre trabajadores y empresarios, centrándose en las exigencias salariales nominales que los últimos hacen a los primeros, desconociendo muchas veces los efectos negativos que estas exigencias pueden llegar a generar en ellos mismos, como el impacto en el salario real que se ve disminuido por el aumento de precio en los productos de primera necesidad derivando ello en mayor pobreza ya que los ingresos mínimos se ven mermados para cubrir las necesidades más elementales (Casas, 2017), impactando así negativamente la calidad de vida del trabajador y sus familias.

Los trabajadores perciben retribuciones o compensaciones salariales según las actividades que desempeñan, el tipo de organización donde trabajan o la actividad independiente que ejerzan. En este punto quien labora en una organización, grande o Mipyme, se pregunta si recibe la remuneración justa por las actividades que desarrolla dentro de ellas (Bonilla, Cardeño y Cardeño, 2015; Pérez, Hernández, Acosta y Chumaceiro, 2009), y quien labora independiente se pregunta si será mejor trabajar para una organización para obtener una 
mejor remuneración (Castaño, Montoya y Restrepo, 2009), remuneración que no es sólo metálica sino que abarca incluso valores éticos que se comparten dentro de las empresas (Sisiruca \& Salazar, 2014)

En cualquier caso, es a través de la remuneración salarial que se obtiene un sustento económico y se suplen las necesidades, desde las básicas hasta las más elevadas, logrando un nivel en la calidad de vida de las personas, es por ello que se propuso el proyecto de investigación, del cual deriva el presente artículo y que lleva como título "El salario real, nominal y la calidad de vida del trabajador colombiano", ya que normalmente se tiene la creencia que aquellas personas que laboran en la actividad de las confecciones y devengan un salario mínimo, no pueden ofrecerse a sí mismas y a su núcleo familiar, buena calidad de vida.

La hipótesis de esta investigación fue que el $80 \%$ de los trabajadores de la actividad de las confecciones de los estratos 2 y 3 de la comuna 10 de ciudad de Medellín (La Candelaria), trabajan en la informalidad laboral con un salario bajo que no les permite tener una buena calidad de vida.

Para aceptar o rechazar la hipótesis planteada fue necesario ahondar un poco sobre el concepto de salario, determinar sus diferentes categorías y su relación con el precio de los bienes y servicios. Hablar de salario y capacidad de compra no es nada fácil, máxime, cuando para abordar esta categoría, debe tenerse en cuenta que, la misma, está relacionada con muchas otras; como: precios, inflación, impuestos, entre otros; y que las mismas, afectan de forma directa e indirecta la calidad de vida del trabajador.

Es así que para darle respuesta se plantearon dos etapas metodológicas: una primera que permite definir el concepto de salario y algunas de sus categorías, abordadas desde la postura de varios autores y alguna teorías, luego se hace una pequeña reseña histórica de la conformación del salario en Colombia, para llegar a una segunda etapa donde se realiza un breve estudio estadístico a partir de los datos entregados por la Cámara de Comercio de Medellín (2019), por último se muestran los resultados y las conclusiones.

\section{Fundamentos Teóricos}

Según el neoliberalismo la flexibilización de los precios a la baja permitiría mejorar la capacidad de compra del consumidor, y mejorar su calidad de vida, ahora bien, al realizar un análisis documental y bibliográfico se encuentran algunas teorías y la postura de varios autores que sirvieron de apoyo para el desarrollo de esta investigación.

Se inicia este artículo considerando las diferentes categorías que tiene el salario, tomando como punto de referencia la posición de algunos autores y tratadistas que han abordado este concepto. Para muchos trabajadores y personas del común el salario es el pago que recibe el trabajador permitiéndole adquirir del mercado aquello que requiere para vivir, sin embargo, para quienes dedican tiempo a su análisis encuentran en la literatura una variedad de conceptos relacionados con esta categoría que enriquecen su estudio para una mejor comprensión

Autores como Caldas y Triana (1957) que toman la definición del salario como la contraprestación que recibe el trabajador por los servicios prestados, asociando esta categoría a otros conceptos como las bonificaciones y horas extras, lo más importante es que reconocen una nueva categoría de salario diferente al fijo, es decir, para ellos también existe el salario variable, de igual forma definen el aporte del trabajador dentro del proceso de producción como un servicio, esto define el trabajo como el desgate 
de músculos y nervios del obrero al interior del proceso de producción tendrá que ser recompensado.

Desde el punto de vista jurídico, el salario ha sido considerado como la remuneración que se debe al trabajador por sus servicios prestados; pero desde el punto de vista social, su significado es diferente en el sentido que el salario le sirve al trabajador para vivir de forma decorosa, atendiendo las necesidades familiares y sociales propias del entorno en el cual convive.

Por su parte Bonilla (1996) expresa que el salario no es exclusivo de la remuneración en dinero metálico, sino que reconoce otra que es la remuneración en especie; dicha categoría es propia de economías agrícolas o hiperinflacionarias, en las que el salario pierde aceleradamente su poder de compra. Cuando esto es así, el obrero no acepta una contraprestación en billetes ni monedas y tiende más bien a volver a la economía del trueque en la que el efecto de los precios sobre la capacidad de compra es casi nula.

Plazas (2003) ofrece una definición asociada dentro de tres categorías fundamentales, pero haciendo énfasis en la relación obrero- patrono donde debe dejarse claro el tipo de remuneración y expresa textualmente:

El salario es la remuneración ordinaria, fija o variable, que recibe el trabajador como contraprestación directa del servicio, cualquiera que sea la forma o denominación que se adopte. Si la suma y los conceptos no son definidos claramente al comenzar el contrato de trabajo, esto va a ser motivo de discrepancias futuras que es preferible evitar ( $p$. 347).

Auscultando un poco más dentro de los aspectos teóricos y conceptuales, autores como Atehortúa (2006), califica al salario como una contraprestación ordinaria que puede ser pagada en dinero o en especie, este autor coincide con Caldas y Triana (1957), al reconocer el salario como una remuneración ordinaria, además estos autores incorporan como elementos constitutivos del salario las horas extras, las primas y las bonificaciones.

Atehortúa (2006) y Bonilla (1996), dentro de sus planteamientos reconocen la existencia del salario como la garantía que se le da al trabajador medida en términos nominales, sino que también reconocen la existencia de un salario en especie como especie pagada al empleado.

Es necesario recordar que este tipo de pagos también es propio de aquellas economías hiperinflacionarias, en la que los precios de las mercancías no tienen control, todo esto a diferencia de lo que presenta Plazas (2003). Autor que reconoce la existencia de una nueva categoría como lo es el salario variable, además de colocar como requisito fundamental y garantía de esa relación obrero-patrono la existencia de un contrato de trabajo.

Plazas (2003) reconoce que el salario como una categoría debe tomarse como un desembolso que recibe el trabajador por su aporte al proceso de producción, como lo define Marx en su texto El capital, las materias primas, insumos, la maquinaria y el capital dentro del proceso productivo son considerados estériles, no producen nada, ni agregan valor; el único factor de producción que genera valor o agrega valor es el trabajo.

Se define el trabajo como ese desgaste de músculos y nervios del ser humano al interior del proceso de producción; de allí que la contraprestación que recibe el empleado, debe servir no solamente para reponer sus energías, sino para mantener $\mathrm{y}$ ofrecer un adecuado nivel de vida a su núcleo familiar. 
Según Atehortúa (2006), son muchas las teorías existentes que permiten conceptualizar acerca del salario, para esto se han diseñado diferentes metodologías, comenzando desde el mismo momento en que se habla del origen del trabajo y su desarrollo histórico hasta el día de hoy; así mismo la normatividad de cada uno de los países decreta, modifica o elimina el salario como unidad de medida del trabajo.

Ahora bien, ya hablando del salario mínimo la Organización Internacional del Trabajo citado por Galvis y Romero (2014) afirman:

[...] debe entenderse como la suma mínima que deberá pagarse al trabajador por el trabajo o servicios prestados, dentro de un lapso determinado, bajo cualquier forma que sea calculado, por hora o por rendimiento, que no puede ser disminuida, ni por acuerdo individual ni colectivo, que está garantizada por la ley y puede fijarse para cubrir las necesidades mínimas del trabajador y de su familia, tendiendo en consideración las condiciones económicas y sociales de los países (p. 24)

Avanzando en este rastreo documental y bibliográfico sobre las diferentes categorías y evolución del salario, autores como Keynes (1965), aborda una nueva categoría, el salario real desde su "Teoría general del empleo, el interés y dinero". Esta categoría de salario real definida por Keynes, es fundamental ya que es la que mejor define la capacidad de compra y la calidad de vida que puede darse el trabajador con su salario nominal que no es más que el número de lo que recibe en la remuneración. Es decir, el "salario real hace referencia al poder adquisitivo del salario" (Graña y Kennedy, 2008, p. 18).

Desde esta dimensión que aborda Keynes, se demuestra la poca o nula preocupación de los empleados por los efectos que puede tener las variaciones de los precios sobre su salario o su capacidad de compra, es cierto que las agremiaciones sindicales o de trabajadores reducen sus luchas pidiendo tanto al gobierno como a los empleadores unos mayores incrementos de los salarios, reduciendo sus exigencias a la parte nominal y no a la real que es por la que verdaderamente se debe luchar.

Es normal que los trabajadores siempre se preocupen por fijarse en cuál sería el incremento de su salario nominal al final de cada periodo, en este mismo sentido lo que menos le preocupa al empleado es saber o percatarse de las variaciones de los precios y como estos pueden afectar su propia calidad de vida. Como lo expresan Campbell y Barraza (2015) no van más allá de exigir mayores incrementos de los salarios mínimos en términos nominales, dejando de lado lo esencial que es la capacidad de compra, sí el trabajador tuviera algunas nociones básicas de economía entendería que las luchas en favor de un mayor salario nominal harían del salario real algo inocuo porque el empresario terminará por incrementar de alguna manera los precios para cubrir los costos.

Keynes (1965), en sus investigaciones, reconoce el salario real como una categoría de las más importantes, porque es una de las razones de peso que permitirían orientar de forma exitosa las luchas entre los empleadores y los empleados, si estos últimos entendieran que un incremento del salario nominal afecta los costos de producción; y que la única forma que tiene el empleador de recuperar esos sobre costos, es incrementando los precios, entonces, los obreros optarían por pedir un control de precios de los bienes y servicios, para garantizar una mejor calidad de vida. 
Esto significaría para el trabajador que el salario mínimo en términos nominales no debe ser la fórmula para una buena negociación y, mucho menos, un indicador macroeconómico de bienestar y calidad de vida, solamente cuando el salario nominal pueda ser enfrentado con los precios, se podría hablar de una medida de bienestar, como se muestra a través del índice de desarrollo humano.

Al respecto Beker (2011) menciona:

Para Keynes era evidente que las fluctuaciones en el nivel de empleo estaban principalmente correlacionadas con las fluctuaciones en el nivel del producto. Esto le parecía tan evidente, que no creía necesario desarrollar una explicación al respecto... Pequeños cambios en el salario real suelen tener un efecto de segundo orden en los beneficios de las firmas y a menudo son compensados por los costos de transacción de despedir o contratar personal. Por ello es que, si se produce una pequeña disminución en los salarios reales, la demanda agregada de trabajo no va a modificarse (p. 28).

Desde luego, si tenemos en cuenta que las variaciones en los precios estarán de alguna manera afectando el IPC (Índice de Precios al Consumidor) y por ende dichas variaciones se ven reflejadas directamente en la capacidad de compra del salario nominal, cuando aumenta el IPC el trabajador realizará una menor demanda por bienes y servicios, esto afecta la demanda por bienes y servicios y con toda razón el incremento de los inventarios o mercancía no vendidas llevará al empresario a tomar decisiones con respecto al empleo o la contratación de mano de obra, este efecto se podría ver fácilmente en una economía altamente protegida, esto también se puede reafirmar con la postura que presenta Beker (2011) al afirmar:
Keynes era un economista con mentalidad práctica. En este sentido, si bien admitía la existencia de flexibilidad en precios y salarios, era muy escéptico respecto a la flexibilidad a la baja. Por eso insiste en que los salarios reales, en la práctica, pueden reducirse sólo por medio del aumento de los precios de los bienes-salarios, no por la contracción de los salarios nominales. Siendo así, está claro porqué ni siquiera considera la posibilidad de que, por medio de una reducción de los precios nominales, un efecto riqueza significativo en el mercado de bienes, pueda llevar la economía hacia el pleno empleo (p. 29).

Lógicamente, en la práctica está demostrado que los obreros jamás aceptan disminuciones en su salario nominal, teóricamente se dice que el salario nominal tiene o presenta esa rigidez a la baja, pero sí es flexible al alza, como se decía hasta antes de la década de los años 90's, que el trabajador no acepta de ninguna manera una disminución de su salario nominal, sin embargo, con la liberalización comercial se demostró que el salario nominal si es flexible a la baja. La modernización del aparato productivo en las economías de mercado, es la que ha permitido una mayor capacidad de compra por cuanto es un control de precios que lo hace automáticamente el mercado.

Al respecto autores como Kornblihtt Seiffer y Villanova (2014) aseguran que el salario real es uno de los indicadores que permite medir la capacidad de compra del consumidor, esto se demuestra al tener en cuenta que para su cálculo se toma como base el salario nominal, medido en pesos y el índice de precios al consumidor, lo cual estará indicando que cada que se presente un incremento de los precios, el trabajador o consumidor tendrá un menor poder de compra y por el contrario si se presenta una 
disminución de la inflación o los precios se tendrá mayor poder de compra lo que en última instancia beneficia o mejorará la calidad de vida, además una disminución de la inflación permitirá adquirir en el mercado aquello que antes no se podía.

Siguiendo con este análisis Ardila (2003) incorpora la relación del salario y la calidad de vida, mostrando además la relación de esta última con algunos de los aspectos clave que se abordan desde el índice de desarrollo humano:

Cuando se han satisfecho las necesidades básicas de alimento, vivienda, trabajo, salud, se determina el interés por la calidad de vida definiéndola como un estado de satisfacción general, derivado de la realización de las potencialidades de la persona. Posee aspectos subjetivos y aspectos objetivos. Es una sensación subjetiva de bienestar físico, psicológico y social. Incluye como aspectos subjetivos la intimidad, la expresión emocional, la seguridad percibida, la productividad personal y la salud objetiva. Como aspectos objetivos el bienestar material, las relaciones armónicas con el ambiente físico y social y con la comunidad y la salud objetiva percibida (pp. 162-163).

Ardila (2003), tiene una apreciación importante que es quizás el fundamento de esta investigación, relacionar el salario con la calidad de vida o el bienestar del trabajador, resultaría sumamente lógico entender que las preocupaciones del empleado cuando ingresa al puesto de trabajo solo se centran en determinar cuál será su pago en términos monetarios, pero esto no debería ser así, sería sumamente interesante esperar que este también se preocupara por que dicha contraprestación le permitiera a él y su familia un bienestar físico y mental; pero en la realidad esto no se presenta de esta manera.
Hablando de calidad autores como Hernández, Chumaceiro, Ziritt y Acurero (2018) expresan que es un cúmulo de sistemas que deben funcionar de manera eficiente para garantizar bienestar colectivo, que incluye oportunidades de generación de ingresos, de empleos de calidad, En tal sentido, Reyes, Hernández, Chumaceiro y Cadrazco (2016) señalan que el término calidad de vida según la OMS está relacionado con la salud y es "el completo bienestar físico mental y social" (p. 64), por su parte Chumaceiro, Hernández y Chirinos (2016) agregan a los conceptos anteriores que la calidad de vida va referida también a derechos económicos, políticos y sociales.

Por su parte Galván (2017), señala que:

El concepto de calidad de vida y bienestar social puede tener cinco campos a comprender como son: físico: donde puede tener aspectos de salud y seguridad física, el material: contar con una vivienda digna y propia, con acceso a todos los servicios básicos, alimentos al alcance, medios de transporte, educativos: existencia y cercanía a una educación donde permita ampliar los conocimientos de forma individual y colectiva, emocional: campo muy importante para cualquier ser humano, mediante el cual permite que su estado emocional vaya creciendo e interrelacionarse eficazmente. Social: donde al ser humano tiene opciones interpersonales con núcleos básicos como la familia y círculos de amistades que permitan desarrollo del mismo (p. 1).

Finalmente, Galván (2017) define de forma más concreta el concepto de calidad de vida, teniendo en cuenta aspectos económicos, sociales y materiales para el ser humano, trabaja esta categoría como algo que lleva a la dignidad de los trabajadores. 


\section{Metodología}

El tipo de estudio realizado tuvo en cuenta aspectos metodológicos cualitativos y cuantitativos, por cuanto en una primera parte se realiza un recorrido bibliográfico y documental que permitió llevar a cabo una aproximación al objeto de estudio de acuerdo a la postura, planteamiento $\mathrm{y}$ teorías llevadas a cabo por varios autores que han tratado el tema, se comienza estudiar las ideas y aportes de Caldas y Triana (1957), Bonilla (1996) hasta llegar a la tesis desarrollada por Keynes (18831946).

Desde el punto de vista cuantitativo fue necesario tener en cuenta para la comprobación de la hipótesis planteada inicialmente, la recolección de datos estadísticos de fuentes confiables como la Cámara de Comercio de Medellín (2019) que permitió obtener la población existente de empresas en la zona, se procedió al diseño del instrumento para la recolección de la información permitiendo la obtención de los datos estadísticos y construir las conclusiones finales.

\section{REsultados}

Después de haber realizado el recorrido bibliográfico y documental, se puede decir que uno de los autores que más se aproxima a realizar una descripción más exacta de calidad de vida es Ardila (2003), cuando en sus análisis, plantea aspectos bien interesantes, cuando afirma que la calidad de vida está estrechamente relacionada con que el trabajador pueda tener acceso a los recursos de subsistencia básicos, los cuales ejercen una gran influencia en su estado emocional y en su equilibrio psicológico.
La carencia o la insatisfacción de algunas de las condiciones mencionadas por el autor, es un síntoma de malestar, que afecta al individuo y a su familia de manera psicológica, y a las relaciones que tienen en su entorno social.

Una vez calculada la muestra estadística y aplicado el instrumento para la recolección de la información, se utilizaron los datos que reposan en la base de datos de la Cámara de Comercio de Medellín (2019), sobre aquellas empresas que en la Comuna 10 están dedicadas a la actividad de las confecciones. Una vez definido y clarificado el concepto de calidad de vida en la encuesta, ésta se aplicó a cien empleados de los estratos 2 y 3 y estos son los resultados obtenidos:

Se observa en la Tabla 1 como en el departamento de Antioquia, existen 4.048 empresas y el $83.12 \%$ se encuentran ubicadas en la ciudad de Medellín, las cuales pertenecen al sector de las prendas de vestir, además, es una de las actividades que más empleos directos e indirectos genera en la ciudad.

TABLA 1.

Confección de prendas de vestir, año 2018

\begin{tabular}{l}
\hline Zona Empresas \\
\hline Antioquia 4.068 \\
Medellín 3.365 \\
\hline
\end{tabular}

Fuente: Elaboración propia a partir de datos de la Cámara de Comercio de Medellín (2019).

Como lo muestra la Tabla 2, en la ciudad de Medellín, de las 3.365 empresas existentes, el $87 \%$ corresponde a microempresarios ubicados en diferentes zonas de la ciudad, el $10.40 \%$ a pequeñas empresas y, tan sólo el 7,21\%, corresponde al tipo de las medianas y grandes empresas. 
TABLA 2.

Sector de las confecciones en Medellín

\begin{tabular}{l}
\hline Nivel Empresas \\
\hline Micro 2.928 \\
Pequeña 360 \\
Mediana 60 \\
Grande 182 \\
Total 3.356 \\
\hline
\end{tabular}

Fuente: Elaboración propia a partir de datos de la Cámara de Comercio de Medellín (2019).

\section{AnÁlisis DE Resultados}

Se tomó una muestra representativa de 100 empleados en la Comuna 10 (La Candelaria) de la ciudad de Medellín, a quienes se les aplicó la encuesta con sus preguntas, y los resultados fueron como se muestra en las siguientes figuras:

Una de las preguntas se enfocó en determinar cuántas personas conforman el hogar del encuestado. Como se ve en la Figura 1 el $57 \%$ entre 1 y 3 personas, el 38\% entre 4 y 6 personas, y solo un $5 \%$ entre 7 y 9 personas.

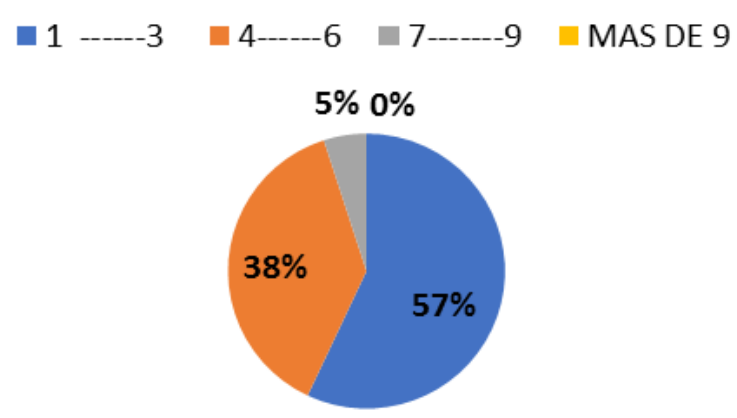

Figura 1. Personas que conforman el hogar

Fuente: Elaboración propia.

Es necesario tener en cuenta como la mayoría de las veces para dar un buen nivel de vida medido en términos de salarios, las cargas al interior del hogar están soportada por los aportes que hacen otros miembros de la familia, es así que se preguntó además del ingreso del empleado de las confecciones, quién más labora en el hogar, las respuestas fueron las siguientes, de acuerdo a la Figura 2: El 52\% de los encuestados responde que hay 2 personas que trabajan en el hogar, seguido de un $24 \%$ que expresan que, en la constitución del hogar, se encuentran trabajando entre 3 y 4 personas. Por último, 21\% para los que solo trabaja 1 sola persona y $3 \%$ que trabajan más de 5 personas.

Por lo cual se demuestra como en la gran mayoría de los hogares del sector de las confecciones no dependen únicamente del ingreso que percibe la persona que se dedica a la actividad de las confecciones y que para obtener un buen nivel de vida es necesario que otros miembros del hogar hagan su aporte.

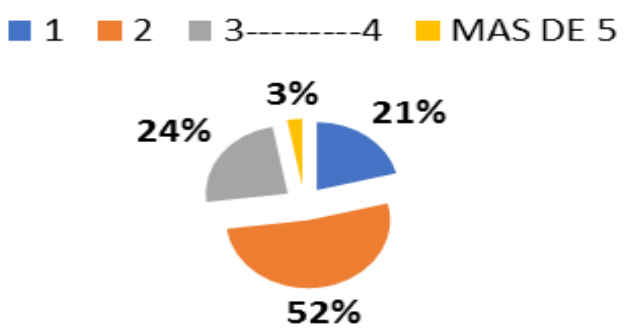

Figura 2. Personas trabajan en el hogar Fuente: Elaboración propia.

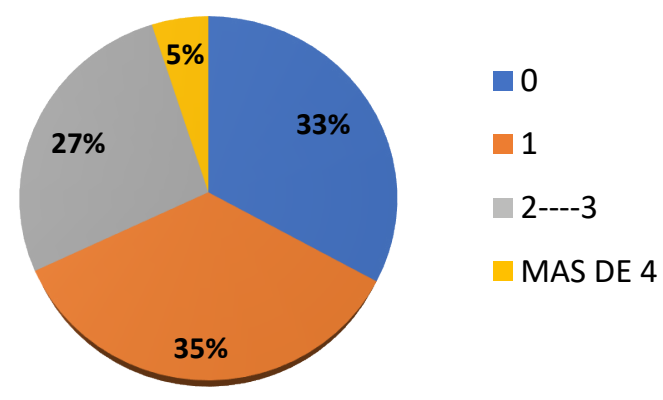

Figura 3. Personas que dependen del trabajador

Fuente: Elaboración propia.

Como lo define Ardila (2003), la vivienda es un bien material que permite medir las condiciones de calidad de vida de las personas. Una vivienda con todos los servicios públicos puede contribuir a generar un mayor bienestar, mejorar el ambiente de habitabilidad y convivencia, y lo más importante, aporta una gran estabilidad emocional y sicológica. 
Se interrogó además a los encuestados si la vivienda en la que vivían era propia o alquilada. Y según muestra la Figura 4 el $64 \%$ paga arrendamiento mensual, mientras el 36\% tiene vivienda propia o familiar. El resultado no deja de sorprender, por el gran porcentaje de salario devengado que es destinado al pago de arriendo. Pero las anteriores preguntas, ratifican como para poder acceder a este arriendo y tener unas adecuadas condiciones de vida, varios miembros del núcleo familiar laboran. Relacionado con lo anterior, se analizó, además, el monto de los ingresos de cada uno de estos estratos.

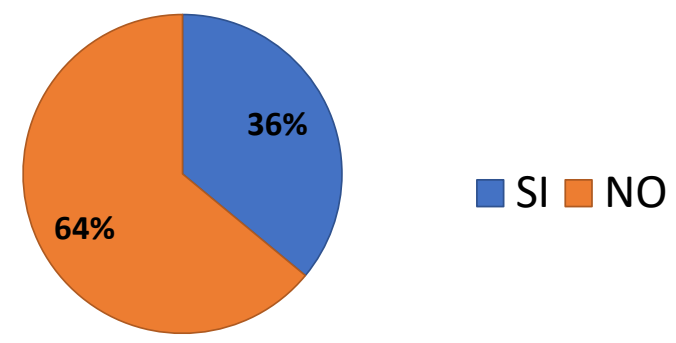

Figura 4. ¿Tiene vivienda propia?

Fuente: Elaboración propia.

Otra de las preguntas se enfocó en determinar el monto de salario que devengan los empleados. La Figura 5 refleja los resultados obtenidos, con un $44 \%$ devengando un salario mínimo legal vigente, otro $44 \%$ más del salario mínimo, y tan sólo un $12 \%$ recibe menos de uno mínimo. Lo anterior demuestra como para el sostenimiento del hogar, tienen un empleo estable, que les garantiza un buen salario. Pareciendo una remuneración acorde con la labor desempeñada.

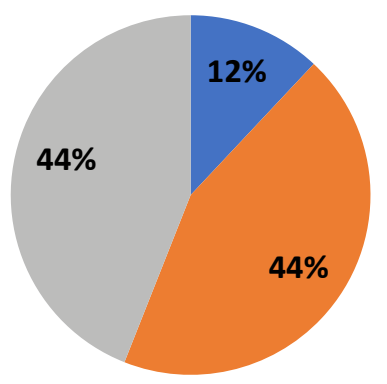

- MENOS 1 SMLV

1 SMLV

$\square$ MAS 1 SMLV

Figura 5. Monto de ingresos mensual del núcleo familiar

Fuente: Elaboración propia.
En la siguiente pregunta se indaga por la afiliación al sistema de salud, en cuanto la salud es considerada un gran complemento para el bienestar del trabajador y su familia, y según la Figura 6 el 83\% tiene una afiliación a una EPS, bien dentro del régimen contributivo o el régimen subsidiado, el 16\% no están afiliados a ningún régimen y el 1\% no responde.

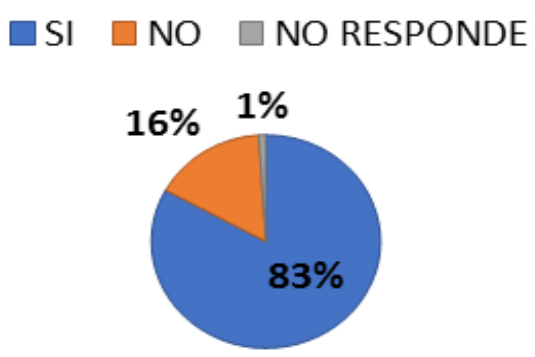

Figura 6. ¿Se encuentra afiliado a la Seguridad social?

Fuente: Elaboración propia.

Grado de escolaridad. Al indagar sobre este aspecto se encontró que el 39\% de los empleados terminaron sus estudios de secundaria, el 19\% un programa técnico, el 19\% una carrera profesional, el 12\% la educación secundaria y un $11 \%$ no alcanzaron a terminar los estudios de secundaria.
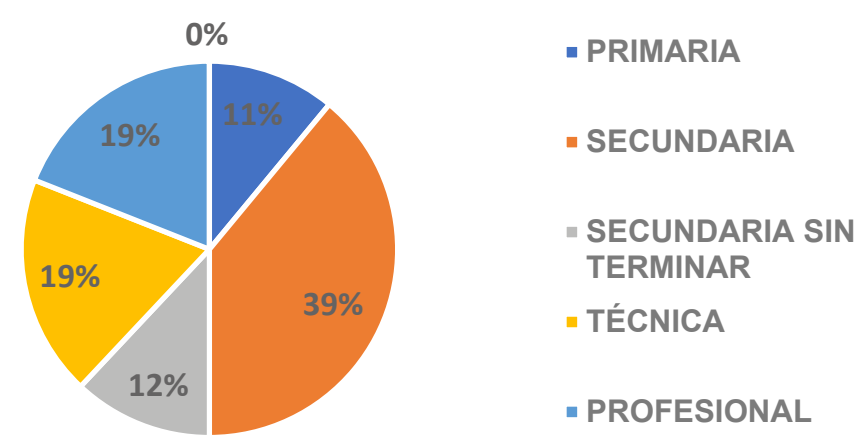

Figura 7. Grado de escolaridad del empleado Fuente: Elaboración propia.

En la siguiente se enfocó en el proceso de la tercerización, las respuestas dadas sobre esta pregunta se resumen así: El 63\% han trabajado en procesos de tercerización y el $37 \%$ indican no tener relación con este tipo de actividad. 


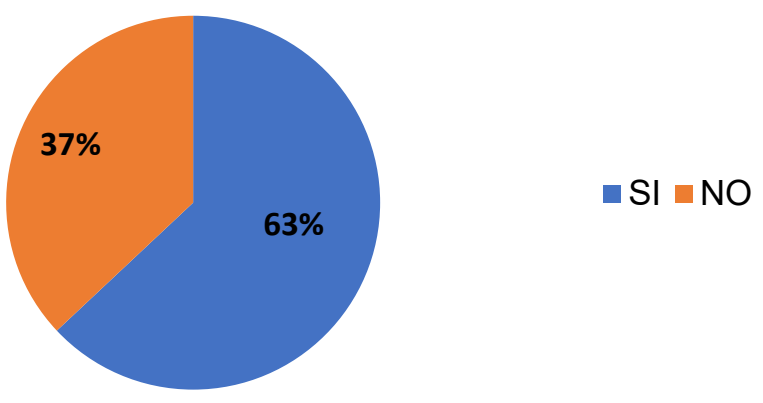

Figura 8. ¿Ha trabajado en tercerización? Fuente: Elaboración propia.

La siguiente pregunta realizada indagaba por el sistema de transporte que utiliza el empleado para desplazarse. El $63 \%$ utilizan el autobús, que es lo más tradicional en las grandes ciudades, y el $37 \%$ utilizan el servicio masivo de transporte metro, como se puede ver en la Figura 9.

$$
\text { - BUS METRO }
$$

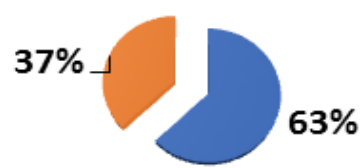

Figura 9. Medios de transporte más utilizados

Fuente: Elaboración propia.

Cuando se les preguntó en relación al subsidio de transporte las respuestas se consolidan en los siguientes resultados. Según la Figura 10 el 53\% no reciben el auxilio de transporte por parte de la empresa, el $32 \%$ recibe el auxilio de transporte y el $15 \%$ no responde
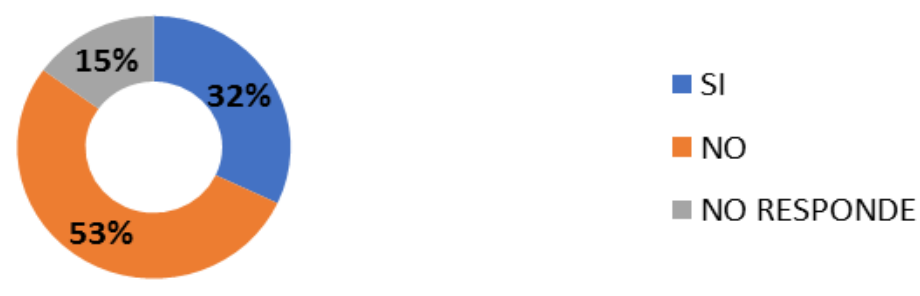

Figura 10. ¿Cuenta con auxilio de transporte por parte de la empresa?

Fuente: Elaboración propia.
Preguntas sobre dotación de servicios públicos. En cuanto a los servicios públicos de la vivienda, y según la Figura 11: El 53\% tienen todos los servicios públicos; el $22 \%$ a por lo menos 5; el $18 \%$ a por lo menos $4, \mathrm{y}$, por último, el $7 \%$ a solo 3. Según los resultados, estos empleados, indica esto que el mayor acceso a los servicios públicos permite que los empleados tengan unas mejores condiciones de vida, según los resultados plasmados en la Figura 12 .

$\square$ TODOS LOS SERVICIOS
5 SERVICIOS
4 SERVICIOS
3 SERVICIOS

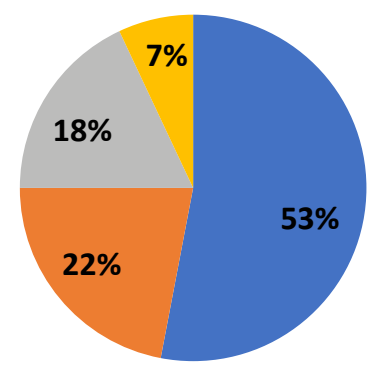

Figura 11. ¿Su vivienda en la actualidad cuentas con cuales servicios públicos domiciliarios teléfono, energía, acueducto, alcantarillado, gas, internet?

Fuente: Elaboración propia.

En el cuestionario se les pregunta: ¿Qué piensan de su calidad de vida? Los resultados entregados, en la Figura 12 permiten concluir como el $92 \%$ expresan que tienen una buena calidad de vida y solo un $8 \%$ indica que su salario no les permite una buena calidad de vida.

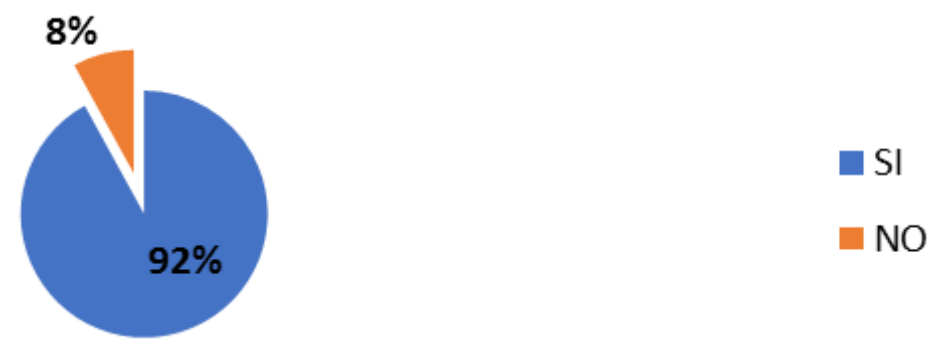

Figura 12. ¿Siente usted que tiene una buena calidad de vida?

Fuente: Elaboración propia. 


\section{Conclusiones}

Una vez interpretado el análisis y los resultados obtenidos de la investigación, se puede afirmar: Como conclusión principal de esta investigación, se rechaza la hipótesis inicial, ya que, según la encuesta aplicada, el $92 \%$ de las personas que trabajan en la actividad de las confecciones de los estratos 2 y 3 de la Comuna 10 de Medellín, afirman tener una buena calidad de vida.

Igualmente, se puede ver como la informalidad laboral no tiene en cuenta las normas laborales que deben proteger a un trabajador, pero paradójicamente, hay una estabilidad laboral, ya que el $39 \%$ de los trabajadores de este sector, llevan más de 10 años laborando en esta actividad.

En esta investigación, se tuvo lugar para escuchar las inconformidades que tienen los trabajadores de la actividad de las confecciones, como es la importación de mercancías chinas, lo cual ha generado menos ingreso ya que, su costo es muy bajo, y no permite que haya una competitividad. Por lo cual, solicitan al gobierno, salvaguardias al producto nacional, para la supervivencia de las microempresas.

Así mismo, se demuestra estadísticamente, como la tercerización con un $63 \%$ de la muestra, ha influido en el sector de las confecciones, puesto que es una opción estratégica de las microempresas, como alternativa de mejorar los costos de producción y seguir siendo competitivos en el mercado.

El salario de los trabajadores es una variable clave que, permite a los trabajadores de la actividad de las confecciones, tener una buena calidad de vida. Sin embargo, es necesario desarrollar una serie de campañas en las empresas, para que tanto empresarios como empleados vean desde su perspectiva, que los incrementos del salario nominal, son una espiral que produce menos capacidad de compra, afectando la calidad de vida del trabajador, de igual forma, unos mayores salarios afectan la capacidad competitiva de las mercancías.

\section{RECOMENDACIONES}

Después de haber realizado esta importante investigación y demostrar por medio de las fuentes primarias que son los propios trabajadores de las confecciones, es recomendable llevar a cabo un estudio muy juicioso sobre los problemas que está causando en la calidad de vida de los trabajadores la tercerización de los procesos de producción que se viene presentando no solo en esta actividad sino en muchas otras, es necesario entender que el proceso aperturista y la entrega de una gran magnitud de mercancía proveniente de países como la China, han llevado a que los empresarios Colombianos busquen mecanismo que les permita enfrentar esta competencia y para ello se han valido de estrategias como la tercerización, que no solo les permite ahorrar costos con el fin de competir, sino que precarizan la calidad de vida de los trabajadores, lo que influye en su calidad de vida y en la calidad de los puestos de trabajo que se ofrecen.

\section{REFERENCIAS}

Ardila, R. (2003). Calidad de vida: una definición integradora. Revista latinoamericana de psicología, 35(2), 161164.

Atehortúa, M. (2006). Manual de legislación laboral. Mexico, D.F.: Nuevo Milenio.

Beker, V. (2011). La teoría macroeconómica y la crisis. Revista de la Facultad de Ciencias Económicas-ChacoArgentina, 7(1), 21-37. http://dx.doi. org/10.30972/rfce.071157 
Bonilla, M. G. (1996). Salarios, precios y productividad. Una relación siempre cambiante. Bogotá D.C.: Fescol.

Bonilla, E., Cardeño, E. \& Cardeño, N. (2015). La función financiera en las micros, pequeñas y medianas empresas, del municipio de Riohacha. Económicas CUC, 36(2), 137-146.

Caldas, T. y Triana, F. (1957). Régimen Laboral Colombiano. Bogotá D.C.: Legis.

Cámara de Comercio de Medellín. (2019). Caracterización económica y empresarial de la comuna 10 la Candelaria. Revista Antioqueña de Economía y Desarrollo. RAED, 21, 8-103.

Casas, J. (2017). Implicaciones de los acuerdos del Fondo Monetario Internacional sobre la pobreza en Colombia. Económicas CUC, 38(1), 9-36. http://dx.doi. org/10.17981/econcuc.38.1.01

Castaño, J., Montoya, L. \& Restrepo, L. (2009). Compensacion salarial y calidad de vida. Scientia et Technica, 15 (43), 89-94

Campbell, U. y Barraza, J. (2015). La crítica de la teoría del valor en Marx, Sraffa y Keynes: un complemento conceptual para la economía. Nicolaita de Estudios Económicos, 10(1), 53-70.

Chumaceiro, A., Hernández, J. y Chirinos, E. (2016). Responsabilidad social universitaria, desarrollo sostenible y ciudadanía ambiental. Cuadernos de RSO, 4(1), 53-64. Disponible en https://ucu.edu.uy/sites/default/files/ facultad/fce/rso/cuadernos-de-rso-vol4-n1-chumaceiro.pdf

Galván, M. (2017). ¿Qué es calidad de vida? Logos Boletín Científico de la Escuela Preparatoria, 1(2). [Online]. Disponible en https://www.uaeh.edu.mx/scige/ boletin/prepa2/n2/m2.html

Galvis, J. y Romero, A. (2014). Salario mínimo legal y salario real: estudio de caso para el eje cafetero. Gestión y región, (17), 19-38.
Graña, J., y Kennedy, D. (2008). Salario real, costo laboral y productividad Argentina 1947-2006. Documentos de trabajo, (12), 1-40. Disponible en http://209.177.156.169/libreria_cm/archivos/pdf_981.pdf

Hernández, J. y Chumaceiro, A. (2018). Acercamiento histórico a la participación ciudadana en Venezuela modelo de relación Estado-Sociedad (19582012). Revista de Ciencias SocialesRCS, 24(2), 56-67

Hernández, J., Chumaceiro, A. Ziritt, G. y Acurero, M. (2018). Cultura para la paz en Colombia. Una aproximación desde las políticas públicas. Opción, 34(86), 612-641

Keynes, J. (1965). Teoría general de la ocupación, el interés y el dinero. México, D.F.: Fondo de Cultura Económica.

Kornblihtt, J., Seiffer, T. y Villanova, N. (2014). De la caída relativa a la caída absoluta del salario real en Argentina. Guillermo de Ockham, 12(2), 41-50.

Pérez, M. E., Hernández, J., Acosta, I. y Chumaceiro, A. (2009). Consideraciones teóricas para el análisis de las Pequeñas y Medianas Empresas como fuente de generación de empleo y su correspondencia ética con la Sociedad. Revista de Formación Gerencial, 8(2), 272-297.

Plazas, G. (2003). La nueva práctica laboral. Bogotá, D.C.: Germán Plazas G.

Reyes, I., Hernández, J., Chumaceiro, A. y Cadrazco, C. (2016). Epilepsia un abordaje social: experiencia de sensibilización y concientización ciudadana. Revista Orbis, 12(35), 58-76. Consultado de http://ojs.revistaorbis.org.ve/ index.php/orbis/article/view/33

Sisiruca, M. \& Salazar, C. (2014). Valores éticos de la responsabilidad social interna en centros de producción audiovisual. Económicas CUC, 35(1), 79-90. 
Villasmil, M. y Crissien, T. (2015). Cambio de paradigma en la gestión universitaria basado en la teoría y praxis de la reingeniería, Económicas CUC, 36(1), 245-274. http://dx.doi.org/10.17981/ econcuc.36.1.2015.29

\section{Biodata}

John Freddy Castro Álvarez es Economista con Especialización en Economía del sector público de la Universidad Autónoma Latinoamericana de Medellín (Colombia). Magister en gestión de las Organizaciones de la U.C.C. Docente-investigador y coordinador de investigaciones de la Corporación Universitaria U de Colombia (Colombia). https://orcid.org/0000-0003-1836-3988

Claudia Marcela Londoño Atehortúa es egresada del programa de Contaduria pública de la Corporación Universitaria U de Colombia. Auxiliar de investigaciones por dos años. En la actualidad trabaja para la Arquidiócesis de Medellín (Colombia). 